\title{
Composite Observer-Based Adaptive Dynamic Surface Control for Fractional-Order Nonlinear Systems with Input Saturation
}

\author{
Bai Zhiye ${ }^{1}$, Li Shenggang ${ }^{1}$, and Liu Heng ${ }^{2}$ \\ ${ }^{1}$ Shaanxi Normal University \\ ${ }^{2}$ Southeast University
}

January 7, 2022

\begin{abstract}
This article proposes an adaptive neural output feedback control scheme in combination with state and disturbance observers for uncertain fractional-order nonlinear systems containing unknown external disturbance, input saturation and immeasurable state. The radial basis function neural network (RBFNN) approximation is used to estimate unknown nonlinear function, and a state observer as well as a fractional-order disturbance observer is developed simultaneously by using the approximation output of the RBFNN to estimate immeasurable states and unknown compounded disturbances, respectively. Then, a fractionalorder auxiliary system is constructed to compensate the effects caused by the saturated input. In addition, by introducing a dynamic surface control strategy, the tedious analytic computation of time derivatives of virtual control laws in the conventional backstepping method is avoided. The proposed method guarantees that the boundness of all signals in the closed loop system and the tracking errors converge to a small neighbourhood around the origin. Finally, two examples are provided to verify the effectiveness of the proposed control method.
\end{abstract}

\section{Hosted file}

DSC.pdf available at https://authorea.com/users/454435/articles/552050-composite-observerbased-adaptive-dynamic-surface-control-for-fractional-order-nonlinear-systems-withinput-saturation 